\title{
COMPUTER APPLICATION FOR MASTERING THE TOPO MEMORIZATION SYSTEM
}

\author{
UDC ((004.92+004.332):159.953)
}

\section{Vladimir Stanković, Dušan Nikolić}

University of Niš, Faculty of Electronic Engineering,

Department of Computation and Informatics, Niš, Republic of Serbia

\begin{abstract}
There are lots of various memorization techniques that could help students. Several studies confirm the possibilities of applying such techniques when learning and studying. In this paper the several memorization techniques are firstly described: the chain of association method, the file cabinets method and the topo memorization system. We then describe the computer application we have developed for the topo memorization system. Our application has both parts for explaining the system, as well as for training. That way it helps the user to adopt and master the topo system, which may be used as an efficient memorization tool by students, as well as by ordinary people.
\end{abstract}

Key words: memorization techniques, topo memorization system, computer application, education, learning

\section{INTRODUCTION}

In order to obtain proper education, students generally have to memorize vast amount of data. In lots of cases the material that is to be memorized is abstract, which makes it difficult to memorize. There are lots of various memorization techniques which may be helpful in such situations. The effectiveness of such techniques is confirmed by a number of studies. Several studies also show that using computers when studying is more efficient, as well as more favored by students, compared to classical studying 'on paper'. This indicates that students would probably be more interested in learning about and practicing memorization techniques with the use of proper computer applications. Speaking about memorization techniques, we should mention mnemonics. They represent any learning techniques (or devices) that aid information retention, by 'translating' information into forms that our brains can retain better than the original forms. Mnemonics are based on the fact that the human mind more easily memorizes spatial, personal, surprising, strange,

Received March 26, 2017

Corresponding author: Vladimir Stankovic

University of Nis, Faculty of Electronic Engineering, Aleksandra Medvedeva 14, 18000 Niš, Republic of Serbia

E-mail: vladimir.stankovic@elfak.ni.ac.rs 
comic or otherwise 'relatable' information, rather than more abstract or impersonal forms of information. The most famous examples of mnemonics are rhymes and acronyms. An interesting example for memorizing the first 7 digits of the number $\mathrm{Pi}$ (3.141592) is the sentence "How I wish I could calculate pi". In this sentence each word corresponds to a proper digit, and the digit is represented by the number of letters of the corresponding word.

Even though there are lots of memorization techniques, which allow everyone to easily and efficiently memorize all types of data [1-7], students have almost no knowledge about their existence. The effectiveness of these techniques has been researched by several authors in various fields [8-13]. This paper represents an attempt of the authors to contribute to the popularization of the memorization techniques, mainly those described in [1]. For one of the methods, called topo memorization system, we developed a computer application that helps the user to adopt the mentioned technique. In our previous work [14], we described the basic version of this application. In this paper we describe three new parts we have developed - a part for the advanced version of the topo system, and two parts intended for training - one for the basic, and the other for the advanced version of the topo memorization system.

The paper is organized as follows. In Section 2 we consider related work. In Section 3 we get to know the basic association technique, which represents a basis for lots of other techniques and methods. Two such methods are the chain of association method and the file cabinets method, which are respectively described in Sections 4 and 5. Sections 6 and 7 describe the topo memorization system and the developed application for its adopting. Section 8 is the conclusion.

\section{RELATED WORK}

There is plenty of information about various memorization techniques. One comprehensive source of such techniques is [1]. Tony Buzan [2-4], founder of the World Memory Championships and the World Championships of the Brain, is an author of several books in this area. His books have been translated into 30 languages. Dominic O'Brien [5], eight times World Memory Champion, is also an author of a few books, in which he teaches common memorization techniques, as well as some techniques that he personally has developed. Ron White [6], an American Memory Champion, has also developed several courses for memorization techniques. Wikipedia is also a source where we can find basic descriptions for many techniques, e.g. [7].

Using various memorization techniques may transform an average person into someone who seems to have supernatural memorization capabilities. That is the reason why several studies have been done on the possibilities of applying memorization techniques in education. We will mention just a few of them. Roediger and Pyc [8] considered strategies, identified by cognitive and educational psychologists, which can improve learning and information retention. They identified three general principles that are inexpensive and have been shown to improve learning (in both laboratory and field experiments): distribution of practice in learning facts and skills, retrieval practice (via self-testing) for durable learning, and explanatory questioning (elaborative interrogation and self-explanation). Milikowski and Elshout [9] studied the difficulty of memorizing numbers between 1 and 100. Their conclusion was that various categories of numbers have different levels of memorizing difficulties. They identified 5 categories: single digit numbers (easiest to memorize), teen 
numbers (10-19), doubled numbers (e.g. 22, 33), large tabled numbers (numbers that factor and therefore appear in the multiplication tables, such as $27,36,45)$, and other numbers that do not fall into any of the previous categories. While memorability for single digit numbers was over $80 \%$, the one for the last category was only around $40 \%$. McCormick and Levin [10] researched the use of the mnemonic keyword method in learning prose. In the experiment, the groups of students that were using the method showed significantly higher levels of recall than the control group. Krzywicka et al. [11] studied the use of IT tools in the laboratory classes to create Buzan's Mind Maps [4] among Material Science course students. The research showed that $76 \%$ of students believed that the use of IT technology in the classroom raises the attractiveness of classes, and $75 \%$ of students would have liked to study the properties and applications of materials using a proper computer program. Noonan [12] also studied the use of Buzan's Mind Maps, but in midwifery education. Gulanowski and Kozak [13] considered if memory training could contribute in learning a foreign language.

Similar to [11], we have also researched the possibilities of using IT tools as a means of learning, mainly for describing and/or practicing memorization techniques. The first author of this paper, in his previous work, has taken part in developing several computer applications that graphically describe the basic association technique, chain of association method and file cabinets method. (These methods will be described in Sections 3, 4 and 5, respectively.) To our knowledge, there are no similar (computer or mobile) applications for these methods, as well as for the topo memorization system (which will be described in Section 6). Memorizing numbers is also a very interesting area. A proper technique for memorizing numbers, which is based on 'translating' numbers into words, is described in $[1,2,3,6,7]$. Each digit may be presented with a consonant and then each 2-digit or 3digit number may be translated into a single word by combining the proper consonants with vowels. We have found several computer/mobile applications that describe/use this technique. Among them, applications [15-18] give help in memorizing numbers - the user enters the number(s) that is(are) to be memorized, and the application shows appropriate words [15-17] or images [18] that may be used for memorizing the entered number(s). In [15] there is also a part in which the user may time oneself while memorizing a series of numbers. Applications [19-22] are developed mainly for practicing memorizing numbers - the user is given a task to memorize a group of numbers in some way. The application [19] also has a part in which text and/or an image may be associated with numbers from 0 to 999 . This part of the application may also be used for the file cabinets method.

\section{BASIC ASSOCIATION TECHNIQUE}

In order to better understand the topo memorization system, which is the main focus in this paper, as well as this memorization system's merits, we will first describe several basic memorization techniques. The basic association technique is one such technique, which represents a basis for many other techniques. It is based on making a connection, i.e. an association between two concepts that are not mutually connected in any way. The simplest way to explain this technique is to use an example. Let us assume that we have the following four pairs of objects: 


$$
\begin{aligned}
& \text { boat - giraffe } \\
& \text { ball - cannon } \\
& \text { cigarette - phone } \\
& \text { skater - bottle }
\end{aligned}
$$

Our task is to memorize these pairs so that when someone mentions one of the words, we should tell the other word in the pair. The basic association technique is based on picturing an image in our heads in which the both objects appear, such that something illogical or impossible or comic is going on, i.e. the picture should contain something very unusual, since that is easily memorized. For example, for the first pair we can imagine a giant giraffe standing in a lake up to its knees in the water and a boat passing below it, like under a huge bridge. For the second pair we can imagine a cannon that is firing a ball instead of a grenade. For the third pair we could imagine a huge amount of cigarettes going out of a phone and filling up the whole room while we are making a call. Finally, for the last pair we could imagine a skater that has bottles instead of skates, making various pirouettes. Each person should imagine what suits them best, and for each person their own picture (movie) will be the best possible picture (movie). It is enough to imagine the scene we are visualizing for only a couple of seconds provided that we imagined something totally unusual.

\section{CHAIN OF ASSOCIATION METHOD}

The basic association technique, explained in the previous section, represents a very powerful technique and lots of other techniques and methods are based on it. The chain of association method directly results from the basic association technique, if we make a chain from the things that are to be memorized. If we got back to the same example from the previous section, we could have the following queue of objects:

$$
\text { boat - giraffe - ball - cannon - cigarette - phone - skater - bottle }
$$

To memorize this queue, we should first use the basic association technique to make an association between the boat and the giraffe, then we should make an association between the giraffe and the ball, then an association between the ball and the cannon etc. until the end of the queue. For the last element in the queue, the bottle, we can do two things: make an association with the first element, the boat, or place it in some weird position. That way we will know that we have come to the end of the queue. A variant of the chain of association method is to make up a funny story in which the elements that are to be memorized are appearing in order in which they should be memorized. The chain of association method can be used for memorizing series of 10, 20, even 50 or more words, such that the whole queue may be reproduced in both directions, forward and backwards (unless the 'funny story' variant is used, in which case one might have difficulties in reproducing the elements backwards). This method is convenient for memorizing the sequence of main points for some lecture one has to give, sequence of chapters of a book, queue of themes when studying for an exam, etc. In [1] it is shown how this technique may be easily adapted for memorizing all sorts of data, not just objects. 
The main advantage of the chain of association method is that there is no limit in the number of elements that can be memorized. We can actually memorize queues that are practically non limited in lengths. On the other hand, the main drawback of this method is that we cannot reproduce the elements out of order.

\section{FILE CABINETS METHOD}

The file cabinets method (also known as the mnemonic peg system [7]) offers a memorizing capability that might appear supernatural to an average person. It enables memorizing lists of elements in such a way that one can easily answer questions such as "Which element is the fifth?", "And which one is the eighth?", "What is the ordinal number of a particular element?" etc. If we used the same example of the queue that begins with the boat and terminates with the bottle, then when using the chain of association method, one has no problem to reproduce the whole queue in both directions, but cannot easily answer the question "Which element is number 5?". To answer that question one would have to go 'through' the first four elements until the fifth one 'comes across'.

The idea in the file cabinets method is to use vocal sound similarities, e.g. one - sun/gun, two - shoe, three - tree, shape similarities, e.g. one - candle/straw, two - swan/duck, three heart, or any other type of similarities for numbers from 1 to $n$, where $n$ is the capacity of the memory buffer one needs - it can be $10,20,50$ or even 100 or more. Different systems use different objects, but the idea is the same - using various types of similarities. For example, TV tower is the object for number 1 in [1] and pencil is the object for the same number in [6]. Obviously, both the TV tower and the pencil are similar to the shape of the number 1 . The object for number 2 in [1] is swan and in [6] is sink. The swan looks like the number 2 when observed from the side and the sink has 2 knobs, it has 2 options - on and off, hot and cold, etc. The object used as number 3 in [1] is triangle, for obvious reasons, etc. How is this used for memorizing the queue? First we make an association (using the basic association technique) between the TV tower (the object assigned to number 1) and the boat (the first element that is to be memorized), then we make an association between the swan (the object assigned to number 2) and the giraffe (the second element that one has to memorize), then an association between the triangle (the object for number 3) and the ball (the third element), etc. until the end of the queue. It is obvious that if we memorized all the elements this way, we would have no problems to tell the exact ordinal number for each of the elements, or to tell each element by its ordinal number, in order or out of order. On the other hand, it is also obvious that we would not have limitless possibilities, like it was the case with the chain of association method - we can only have a memory buffer with capacity that is as large as the number of numbers we have pre-memorized, i.e. 'translated' into proper objects. This means that the file cabinets method has the opposite merits/drawbacks compared to the chain of association method. It allows out of order reproducing (or even memorizing) the elements, but needs pre-memorizing the proper ordinal number elements to get a memory buffer, and the buffer obviously must have a limited size. 


\section{TOPO MEMORIZATION SYSTEM}

The topo memorization system [1] is, in a way, a combination of the methods from previous two sections - the chain of association method and the file cabinets method, in the sense that it has the advantages of both methods. We can memorize lists in a way to be able to tell each element by its ordinal number, as well as to tell the ordinal number for each of the elements, both in order and out of order, and at the same time we can easily form memory buffers with relatively large sizes.

The ordinal numbers in this method are memorized as positions in a room. In the basic version each room has 10 positions and in the advanced version each room has 50 positions. The basic version enumeration is given in Fig. 1. In [1] it is assumed that the entrance (door) of the room is always in the middle of the wall, like it is shown in Fig. 1a. In that case the position where one enters the room is signed as number 1, and the rest of the numbers until 8 are positioned in a clock-wise direction - number 2 is the first corner on the left, the middle of the left wall is number 3 , number 4 is the following corner, etc. until number 8 , which is the last corner. The middle of the floor is signed as number 9 , and the middle of the ceiling (or the chandelier, if there is one) as number 10 . We wondered what one should do in cases where the entrance of the room is ane of the corners, and concluded that the simplest way would be to keep the positions the same, and simply mark he first position where one enters the room as 2 or as 8 , depending on whether the entrance is at the left or at the right corner. This is shown in Fig. 1b and Fig. $1 \mathrm{c}$, respectively. This way the walls are always signed with odd numbers $(1,3,5,7)$ and the corners are always signed with even numbers $(2,4,6,8)$. In that case we are able to use this enumeration with literally any room we have ever seen or been into.

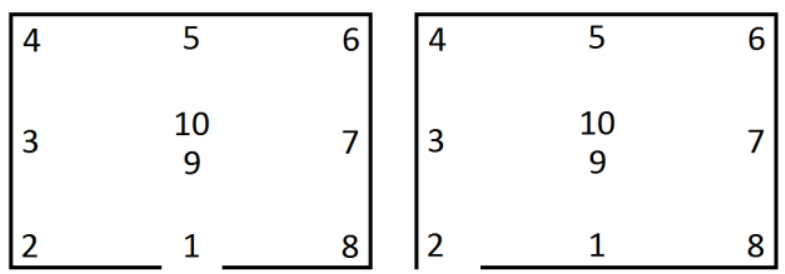

Fig. 1a

Fig. $1 \mathrm{~b}$

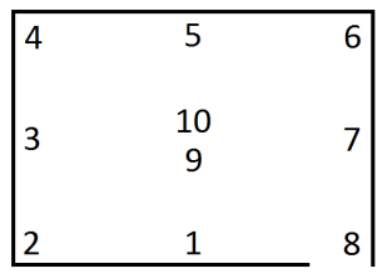

Fig. 1c

Fig. 1 Basic version of the room numeration for the topo memorization system

If we need more than 10 places we can simply use another room (or several rooms, if needed). For example, Fig. 2 shows a plan of a residence with 5 rooms. As each room has 10 
places, we can use this for memorizing a list of 50 elements. This means that by just adopting the basic version of the topo system, we can get a memory buffer with 50 (or more) elements. And that is accomplished by using just one residence with 5 (or more) rooms. We can, of course, use as many apartments/residences as we need.

\begin{tabular}{|c|c|c|c|c|c|c|c|c|}
\hline 4 & 5 & 6 & 12 & 13 & 14 & 44 & 45 & 46 \\
\hline \multirow{2}{*}{3} & 10 & & & 20 & & & 50 & \\
\hline & 9 & 7 & 11 & 19 & 15 & 43 & 49 & $4 /$ \\
\hline \multirow[t]{5}{*}{2} & 1 & 8 & 18 & 17 & 16 & 42 & 41 & 48 \\
\hline & & & 28 & 21 & 22 & 32 & 33 & 34 \\
\hline & & & & 30 & & & 40 & \\
\hline & & & 27 & 29 & 23 & 31 & 39 & 35 \\
\hline & & & 26 & 25 & 24 & 38 & 37 & 36 \\
\hline
\end{tabular}

Fig. 2 Using the basic version for a residence with 5 rooms

The full potential of the topo system is accomplished by using its advanced version, which can be seen in Fig. 3 (which was taken from [1]). Now each room has 50 positions. Each of the 4 walls and the floor has 9 positions, with values 2-10, 12-20, 22-30, 32-40 and 42-50, with a layout that suits the word ' $Z$ ' - the first three values (e.g. 2, 3, 4) are positioned 'at the top' from left to right, the next three values (e.g. 5, 6, 7) are 'in the middle' from right to left, and the last three values (e.g. 8, 9, 10) are 'at the bottom' from left to right. The ceiling has the values $1,11,21,31,41$, which are 'joined' to the proper wall. Number 1 is in the middle of the ceiling, which means that the rest of the numbers of the first decade (2-10) are on the floor. Number 11 is positioned near the wall that is in front of the observer (after entering the room), which means that the rest of the numbers of the second decade (12-20) are positioned on that wall. Number 21 is near the right wall, which means that the third decade is positioned on the right wall. The rest of the numbers are positioned in the same manner, and each subsequent decade is on the next wall, when we move in a clock-wise direction, observed from the ceiling. By using the advanced version of the topo system we can memorize a list of 250 elements, be able to tell the ordinal number for each element or tell each element by its ordinal number, in order or out of order, and all of this can be accomplished by using just one residence with 5 rooms.

An interesting thing to notice is that, to our knowledge, [1] is the only source that describes the topo memorization system. In spite of its huge memorization potentials, neither of the books and courses [2-6] mentions this system. This was one more reason for us to develop an application for adopting this system, and that way help the interested students. 


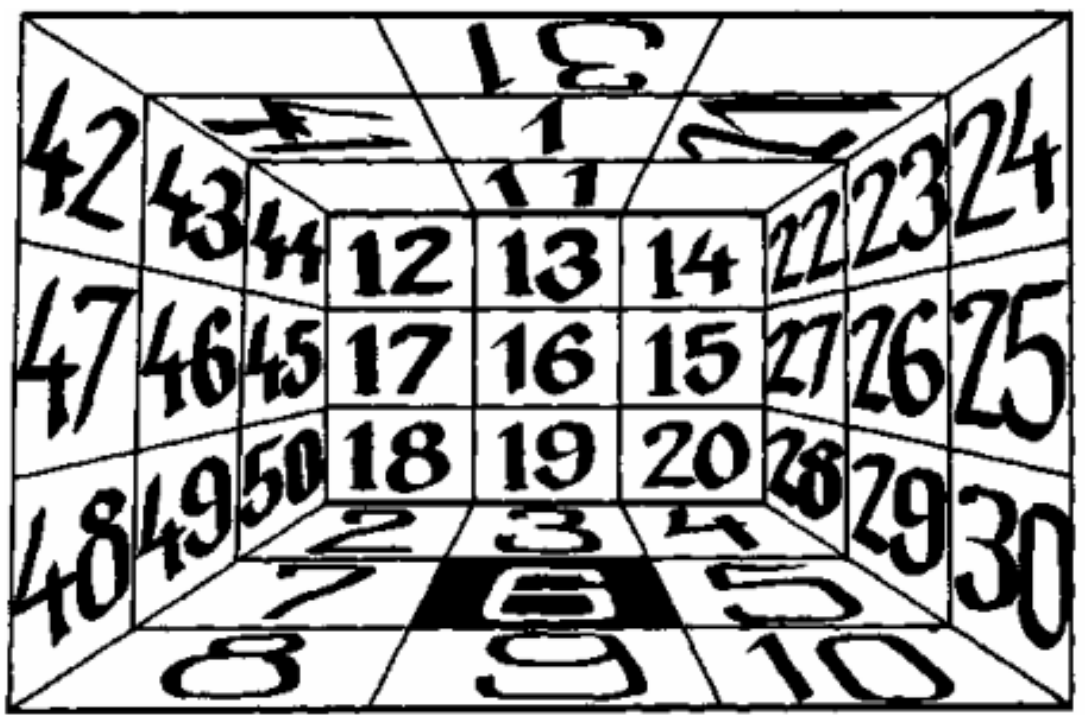

Fig. 3 Advanced version of the room numeration for the topo memorization system

\section{THE APPLICATION}

In this section we will describe the application we have developed for adopting the topo memorization system. The starting window of the application, as can be seen in Fig. 4 , shows two buttons, which help the user to choose whether one is going to work with the basic or the advanced version. In case the basic version is chosen, the application shows the basic enumeration, as can be seen in Fig. 5. We are positioned at the place for number 1 , and we can see the rest of the walls (signed as 3, 5, and 7), and the two corners between them (signed as 4 and 6). We can also see the middle of the floor (signed as 9) and the ceiling (signed as 10). The wall signed as number 1 is behind us, hence we cannot see that wall. We also cannot see the two corners signed as 2 and 8. To ease the process of prememorization, we use different colors for the walls and the corners, as can be seen.

By moving the mouse left or right the application will simulate that we are turning on our left or on our right. That way we can see the wall and the corners that are not visible at the start. We can also move the mouse forward or backward and the application will simulate that we are floating in the air and looking down or that we are lying down and looking up. By all these 'effects' the user gets the feeling that they are moving around the room, which helps them to more easily memorize the proper numeration. Fig. 6 shows several screen shots which partially demonstrate the completeness of the possible 'movements'. 


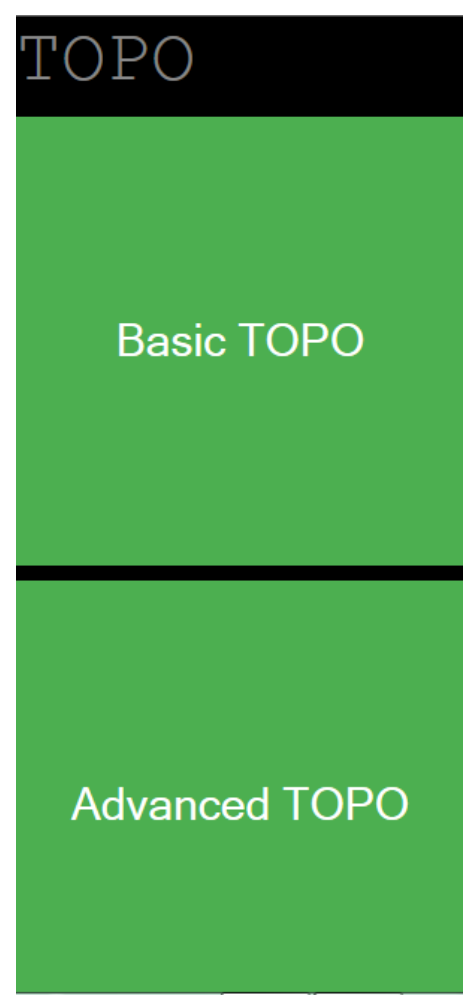

Fig. 4 Part of the starting window of the application

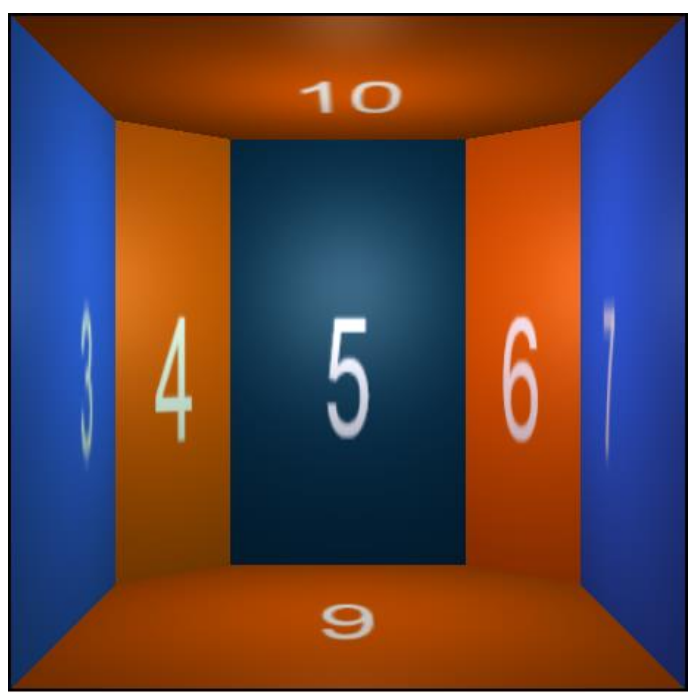

Fig. 5 Starting window of the introduction for the basic version 


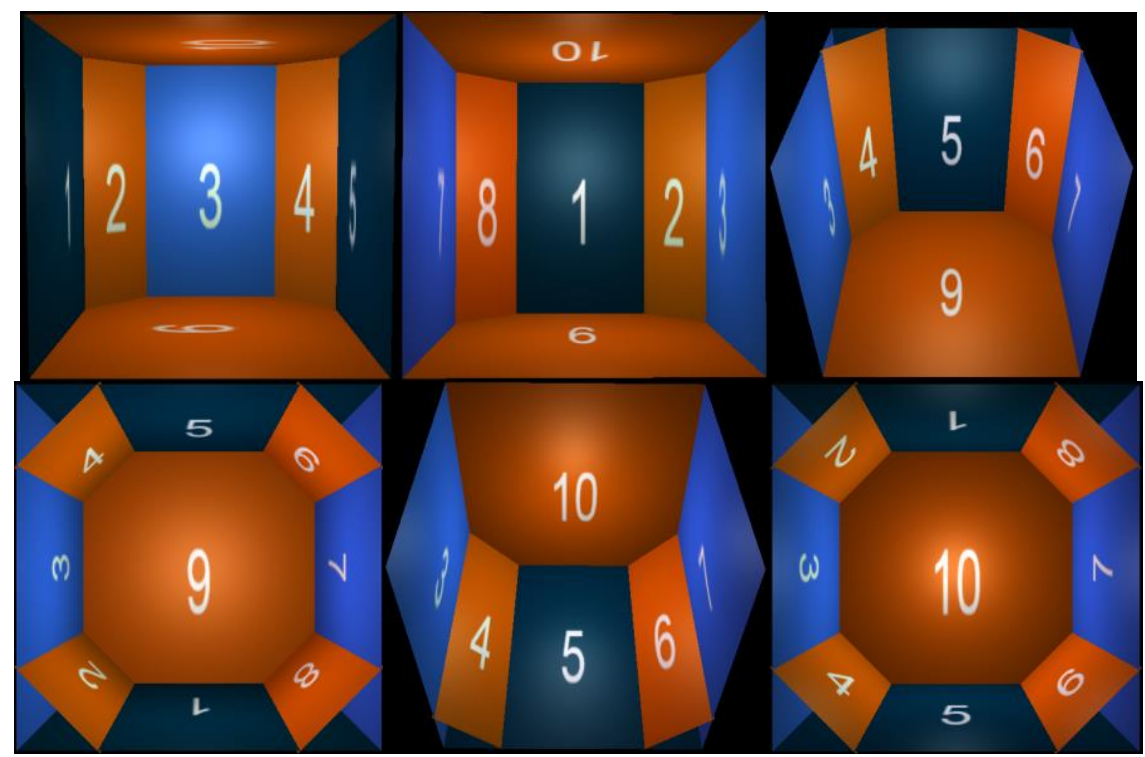

Fig. 6 Several characteristic screen shots of the introduction for the basic version

At the left corner there is a button 'Start Training' (not shown in Figures 5 and 6). Clicking this button will activate the training of the basic version. In the left part of the new window, the user has to enter several options, which are shown in Fig. 7. The pause before and after room rotation is entered in seconds, and the number of steps for room rotation defines for how many steps the room will rotate from starting to finishing position. The starting position is the position shown in Fig. 5, in which we see the wall with number 5 directly in front of us (without showing the numbers). The finishing position is with the wall or corner of the selected number in front of us. Namely, during this phase the application randomly chooses a number between 1 and 10 and shows it on the left part of the screen, below the options. After the pause for 'before room rotation' elapses, the room, which is shown on the right part of the screen, will rotate finishing with the corresponding wall/corner in front of us. After the pause for 'after room rotation' elapses, the starting position of the room will be shown again. Then another number is randomly chosen and everything is repeated. To make things more difficult, during the training phase no numbers are shown in the room, just the walls and the corners. The user can control the training by using the buttons 'Start', 'Stop' and 'Back', with obvious meanings. This part of the application is one of the most important parts, as it trains the user to 'rotate' in the room and that way more easily memorize the proper positions for each of the numbers. This is valuable, as using the topo system is performed in the same way - memorizing and recalling back are both done by proper rotating in the room in one's mind. Several screen shots of the room, during this phase, are shown in Fig. 8. This figure shows the finishing positions for several numbers (each screen shot was taken after the room rotation stopped). The shown 6 screen shots 
respectively correspond to numbers: $7,3,8,4,9$ and 10 . At the center of the room there is an arrow, which shows the starting orientation of the user (towards the wall signed as number 5). This may help some users in their beginning phases of the training.

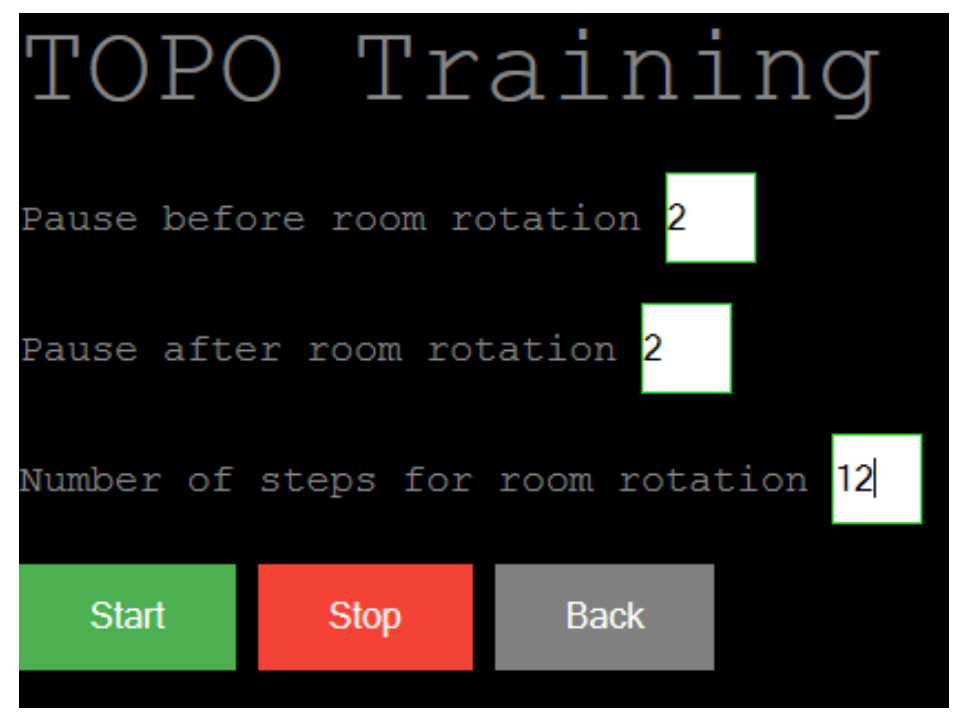

Fig. 7 Options for the training phase

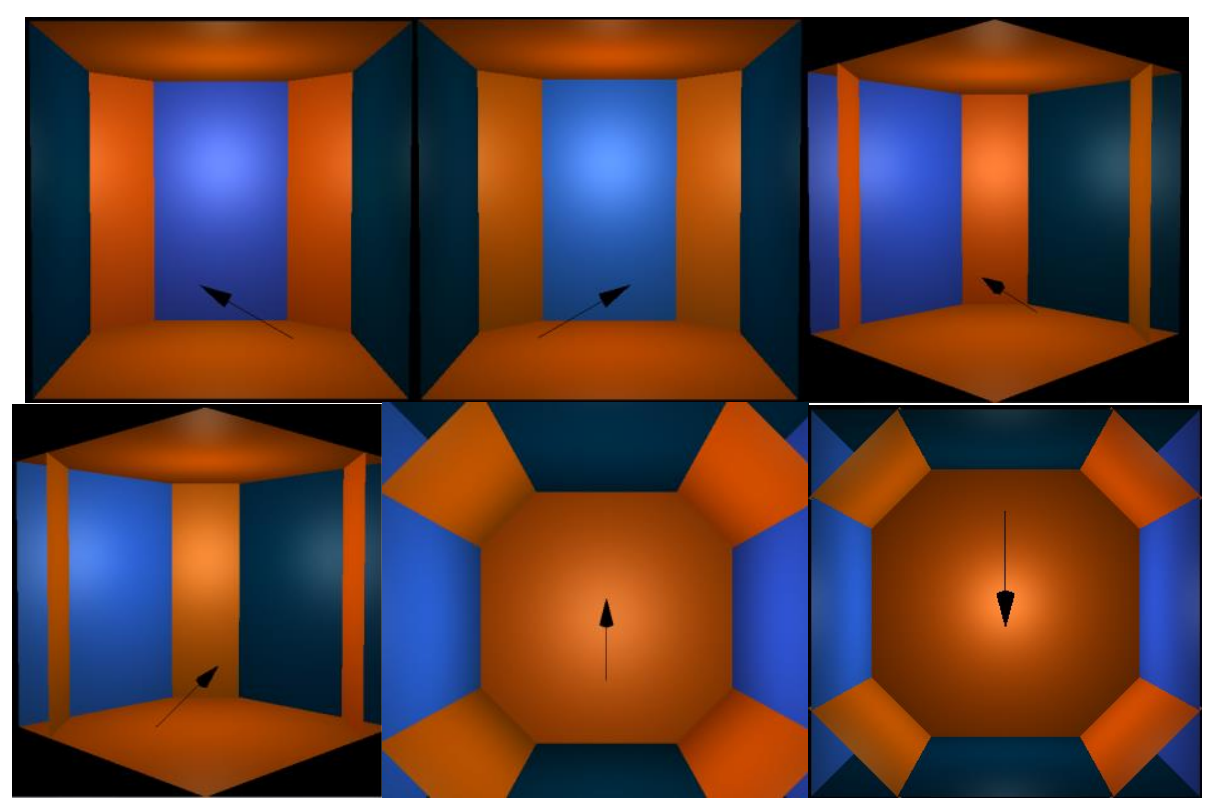

Fig. 8 Several screen shots of the room during the training phase - basic version 


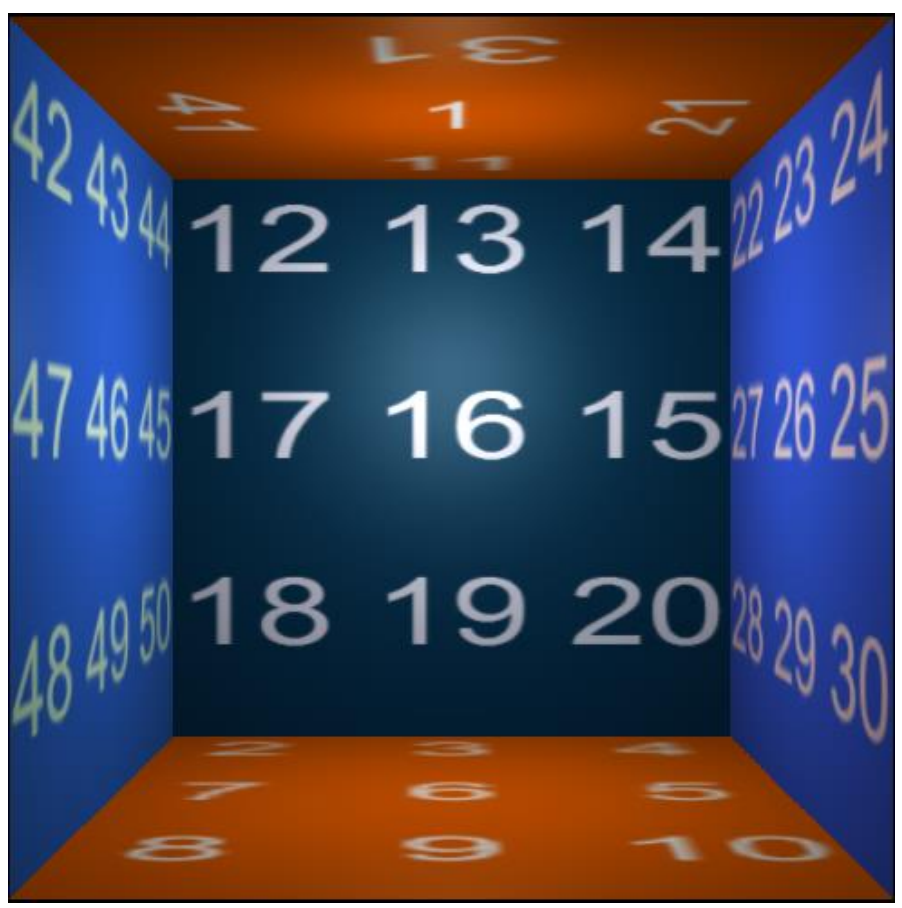

Fig. 9 Starting window of the introduction for the advanced version

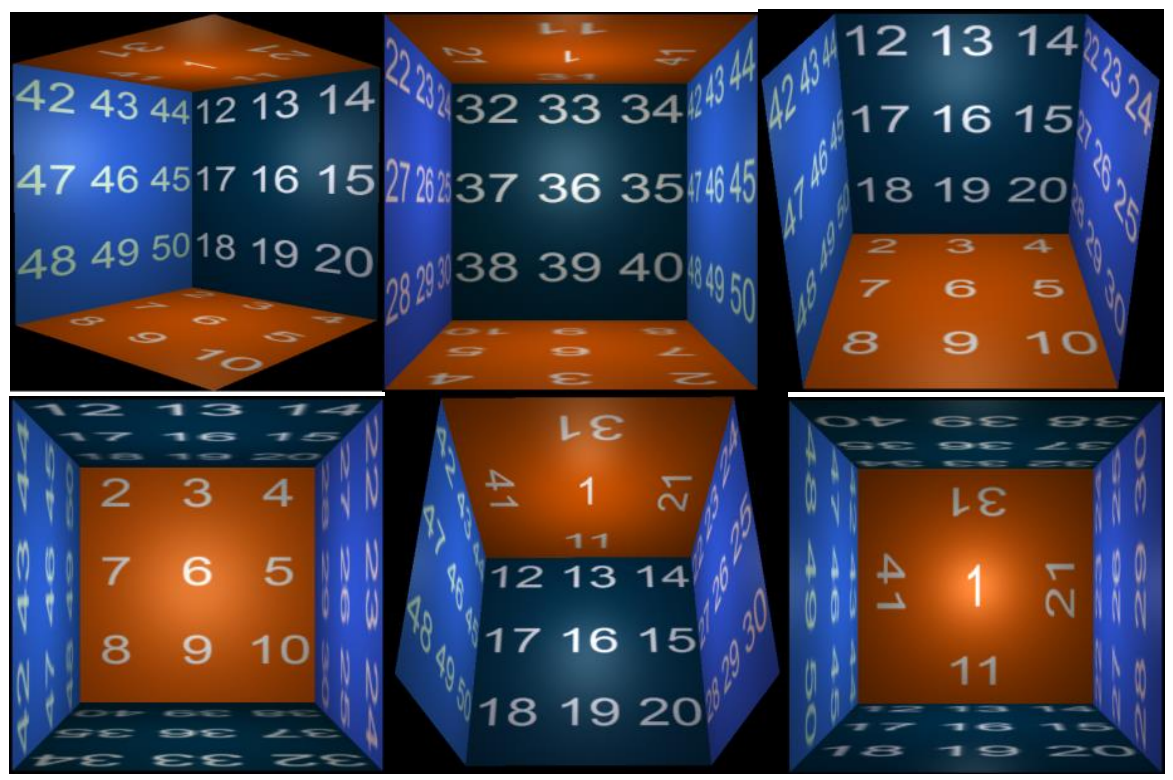

Fig. 10 Several characteristic screen shots of the introduction for the advanced version 
In case the user chooses the advanced version, the starting window will show the advanced enumeration, as shown in Fig. 9. We can see the wall with numbers 12-20 in front of us, the first decade is on the floor, the numbers 22-30 are on the right wall and the numbers 42-50 are on the left wall. The numbers 32-40 are on the wall behind us, therefore we cannot see them and the ceiling has the numbers $1,11,21,31$ and 41 . Similarly to the basic version, we can move the mouse left or right and that way we will 'turn' on our left or our right. We can also move the mouse forward or backward and simulate moving towards the floor (i.e. floating up in the air) or towards the ceiling (i.e. lying down). Fig. 10 shows several characteristic screen shots of the room while we are moving.

Clicking the 'Start Training' button will start the training. The left part of the window will show the same options we had for the basic version training (shown in Fig. 7), and the right part of the window will show the room without the numbers. The training is very similar to the basic version, the application randomly chooses a number between 1 and 50 and shows it at the left part of the window. The room also rotates similarly to the basic version, the only difference is that now it is not enough for the application to just finish with the proper wall/floor/ceiling in front of us - it has to also show the position of the selected number, as 9 numbers are positioned on each wall, as well as the floor. The ceiling also has 5 numbers, from which the proper one should be shown. This is done by using a light rectangle at the proper position, as can be seen in Fig. 11. This figure shows several screen shots during the training. These screen shots are taken in the finishing positions, and they correspond to numbers 50,27, 38, 18, 9 and 11 , respectively.

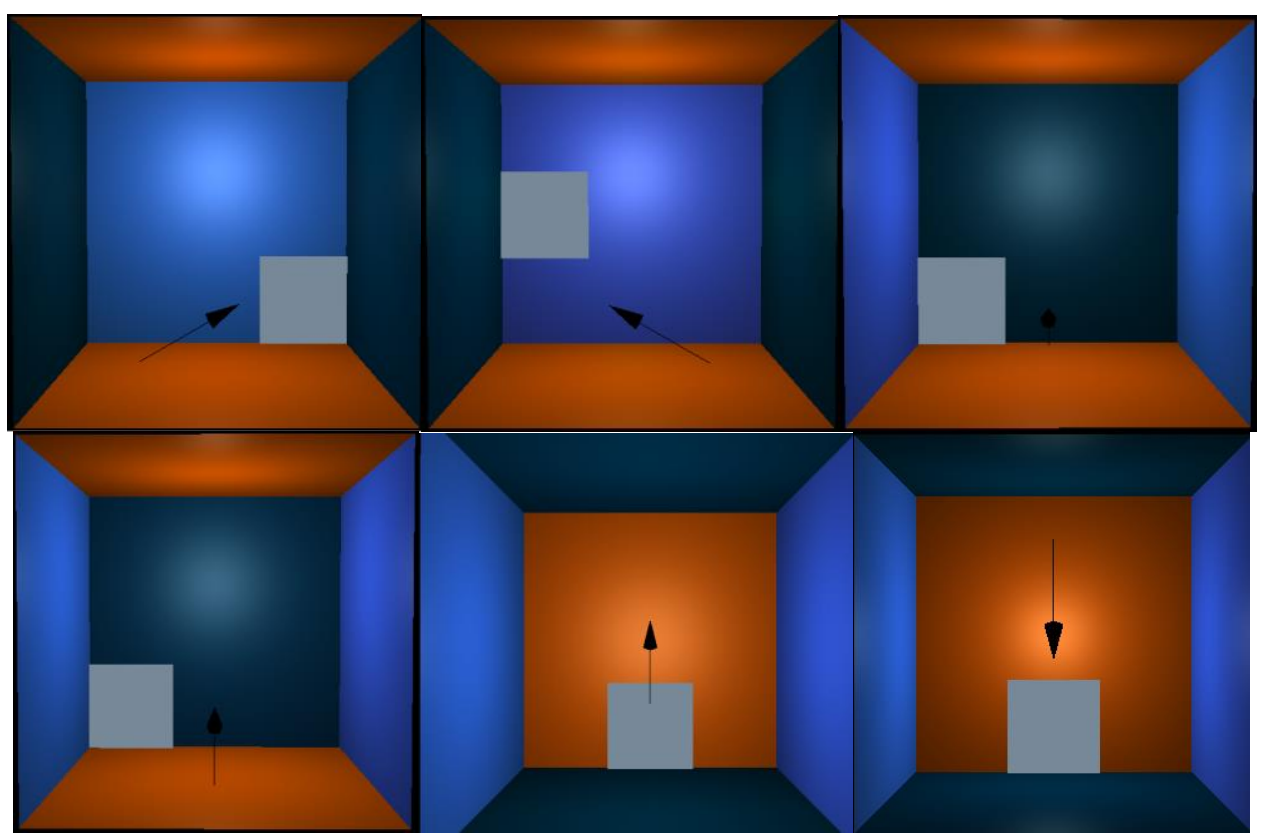

Fig. 11 Several screen shots of the room during the training phase - advanced version 
As we can see, the application practically simulates the process that needs to be done in one's head for the sake of applying the topo memorization technique. The option to define the pause before room rotation gives the user the needed time to think and the option to define the pause after room rotation gives the needed time to check the correct answer, i.e. to compare it with the conceived one, while the number of steps for room rotation practically increases or decreases the room rotation speed. These three options are all valuable, as they give adaptability and the user can define them according to one's needs and current level in the process of mastering the technique. Therefore, the application is suitable for beginners, intermediate users, as well as experts. It is expectable that regular use of the application should contribute to mastering this interesting memorization system.

\section{CONCLUSION}

There are many memorization techniques that allow easy memorizing of all sorts of data. Students, no matter what type of faculty they are studying, have to memorize various types of data. Therefore, they could really benefit from using proper memorization techniques, especially regarding the fact that the effectiveness of such techniques has been proven by many studies. Applying personal computers or mobile devices can also be useful for learning the needed materials, as well as for practicing memorization techniques. That way the process of learning may be significantly relieved.

In this paper we have described the basic association technique and its usage in the chain of association method and the file cabinets method. We have also described the topo memorization system. We saw that this system has the merits of both the chain of association method and the file cabinets method - one can easily form memory buffers with large capacities, which has some similarities with the chain of association method and the memorized data can be reproduced out of order, similarly to the file cabinets method. For the topo memorization system we have developed a proper computer application for both the basic and the advanced version of the system. The application not only introduces the user with the system, but also has parts for training, which makes it useful for complete mastering of this memorization system. We hope that this application will help students and interested readers in their studying, as well as everyday life.

Acknowledgement: The research presented in this paper was supported in part by the Serbian Ministry of Education, Science and Technological Development [TR32012].

\section{REFERENCES}

[1] A. M. Semorie, R. Semorie, S. Popović, I vi možete imati super pamćenje. (Eng: You too can have a super memory). Beograd, 1980.

[2] T. Buzan, "Use your memory", Guild Publishing London, 1986

[3] T. Buzan, "The Memory Book: How to Remember Anything You Want", BBC Active, Educational Publishers LLP, Pearson Education Group, London, 2010.

[4] T. Buzan, B. Buzan, "The Mind Map Book: Unlock Your Creativity, Boost Your Memory, Change Your Life", BBC Active, Educational Publishers LLP, Pearson Education Group, London, 2010.

[5] D. O'Brien, "How to Develop a Brilliant Memory Week by Week: 52 Proven Ways to Enhance Your Memory Skills", Watkins Publishing, 2014. 
[6] R. White, "Memory in a month", audio program, http://www.brainathlete.com/memory-month/

[7] "Mnemonic peg system", Wikipedia, https://en.wikipedia.org/wiki/Mnemonic_peg_system

[8] H. L. Roediger III, M. A. Pyc, "Inexpensive techniques to improve education: Applying cognitive psychology to enhance educational practice", Journal of Applied Research in Memory and Cognition, Vol. 1, Issue 4, December 2012, pp. 242-248.

[9] M. Milikowski, J. J. Elshout, "What makes a number easy to remember?”, British Journal of Psychology, Vol. 86, Issue 4, 1995, pp. 537-547.

[10] C. B. McCormick, J. R. Levin, "A Comparison of Different Prose-Learning Variations of the Mnemonic Keyword Method", American Educational Research Journal, Vol. 21, No. 2, 1984, pp. 379-398.

[11] M. Krzywicka, D. Dobrowolski, J. Grudzinski, "Application of Tools for Creating Maps of Knowledge in Teaching Material Science to Students of University of Life Sciences”, Annales UMCS Informatica, Vol. 15, No. 1, 2015, pp. 16-20.

[12] M. Noonan, "Mind maps: Enhancing midwifery education", Nurse Education Today, Vol. 33, Issue 8, August 2013, pp. 847-852.

[13] J Gulanowski, A. Kozak, "Can memory training positively affect the skills of learning a foreign language and support learning English by older students?", The Journal of Education, Culture, and Society, Issue No. 2, 2012

[14] Vladimir Stankovic, Dusan Nikolic, "Topo Memorization System", The $4^{\text {th }}$ International Virtual Research Conference in Technical Disciplines, October 17. - 21. 2016, pp. 50-54

[15] "Major System: Master Memorizing Numbers 1.0", http://appfinder.lisisoft.com/app/major-systemmaster-memorizing.htm

[16] “010 Memorizer", http://www.sweetscape.com/010memorizer/

[17] K. Dworakowski, "Pinfruit", http://pinfruit.com/

[18] "Memorize iDi", https://play.google.com/store/apps/details?id=com.idicreative.memorizeFree\&hl=en

[19] "Digit Drill”, http://divedeepsoftware.com/digitdrill.html

[20] "Memoriad Competition Simulator", http://www.memoriad.com/memoriadsoftware.asp

[21] "Memorizing numbers Test", https://itunes.apple.com/gb/app/memorizing-numbers-test/id358120098?mt=8

[22] "Memorizing Numbers Game", https://itunes.apple.com/us/app/memorizing-numbers-game/id423290147?mt=8 